\title{
ORIGINAL ARTICLE \\ Posterior only versus combined posterior and anterior approaches for lower lumbar tuberculous spondylitis with neurological deficits in the aged
}

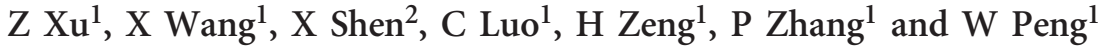

\begin{abstract}
Study design: Retrospective case-control study.
objectives: To analyze the results of two surgical treatments for lower lumbar tuberculous spondylitis with neurological deficits in the aged.

Methods: We studied 33 cases of lower lumbar spinal tuberculous spondylitis treated in our center from January 2006 to October 2010. The cases were divided into two groups: 16 cases (group A) underwent single- or two-stage anterior debridement, bone grafting and posterior instrumentation, and 17 cases (group B) underwent single-stage posterior debridement, decompression, interbody fusion and instrumentation. Clinical and radiographic results were analyzed and compared between the groups.

Results: Patients were followed for a mean of 41.3 months (range 36-48 months). The average operative durations were $276.9 \pm 23.8$ and $193.8 \pm 22.5$ min in groups $A$ and $B$, respectively. The average hospital stay was $18.2 \pm 3.2$ days for group $A$ and 13.4 \pm 1.6 days for group B. Average intraoperative blood loss for groups A and B was $1187.5 \pm 163.0$ and $804.7 \pm 134.1 \mathrm{ml}$, respectively. Operative complications affected four patients in group A and one in group B. Solid fusion occurred at 12 months in the other 32 cases. Neurological status was significantly improved in all cases. Kyphosis was significantly corrected after surgical management, but loss of correction occurred in both groups.

Conclusion: Single-stage posterior debridement, decompression, interbody fusion and instrumentation might be a better surgical treatment than combined posterior and anterior approaches for lower lumbar tuberculous spondylitis with neurological deficits in the aged, offering fewer complications and a better quality of life.
\end{abstract}

Spinal Cord (2015) 53, 482-487; doi:10.1038/sc.2014.252; published online 10 February 2015

\section{INTRODUCTION}

Spinal tuberculosis (TB), the most common type of extrapulmonary $\mathrm{TB}$, is a severe spinal disease that frequently causes kyphotic deformity, neurological deficits and even paralysis. ${ }^{1}$ With the increase in spinal TB incidence and the rising proportion of elderly people in China, spinal TB in elderly patients is on the uptrend, ${ }^{2}$ and it remains a leading cause of paraplegia in developing countries. ${ }^{3}$ Recent advances in the pharmacological treatment of spinal TB and our understanding of drug resistance have changed the management of this disease. However, kyphotic deformity cannot be arrested by conservative treatment, ${ }^{4}$ and neurological recovery is slower with non-surgical treatment and only occurs in a small percentage of cases. ${ }^{5}$ Furthermore, based on a literature review, the results of conservative treatment—especially in elderly populations receiving long courses of anti-TB chemotherapy and extra immobilization-were unsatisfactory on the whole. ${ }^{6}$ Thus surgery is frequently imperative for spinal TB in the aged.

Traditionally, the anterior approach to the spine has been preferred, because TB pathology mainly affects vertebral bodies and disc spaces; the anterior approach enables direct exposure of the pathological site. However, complications such as postoperative ileus, retrograde ejaculation in males and ureteral injury are often associated with an anterior approach. ${ }^{7}$ Additionally, anterior instrumentation is difficult to implement at the L4-L5 and L5-S1 segments because of the special anatomic characteristics and position of these segments. ${ }^{8}$ Combined posterior and anterior procedures are often associated with prolonged operative times, greater blood loss and increased complications, ${ }^{9}$ making them unsuitable for elderly patients in poor health. ${ }^{10}$ To our knowledge, the posterior only approach reported by some surgeons can achieve equally good outcomes as anterior debridement and bone grafting. ${ }^{11}$ In this study, we compared the clinical outcomes of the posterior only approach and the combined posterior and anterior approach for lower lumbar TB with neurological deficits in the aged.

\section{MATERIALS AND METHODS}

\section{Basic information}

Written informed consent was obtained from all patients; the Xiangya Hospital Ethics Committee approved the study protocol. From January 2006 to October 2010, 33 patients diagnosed with lower lumbar TB were enrolled in this study. There were 18 males and 15 females. Their mean age (according to the Chinese standard) at the initial operation was $70.1 \pm 4.6$ years, ranging from 62 to 79

${ }^{1}$ Department of Spine Surgery, The Xiangya Hospital of Central South University, Changsha, Hunan, China and ${ }^{2}$ Department of Spine Surgery, The People's Hospital of Hunan Province, Changsha, Hunan, China

Correspondence: Dr X Wang, Department of Spine Surgery, The Xiangya Hospital of Central South University, 87\# Xiangya Road, Changsha, Hunan 410008, China. E-mail:wxyxydoc@163.com

Received 1 November 2014; revised 18 December 2014; accepted 29 December 2014; published online 10 February 2015 
years (Table 1). In 20 cases, only the disc was involved; other cases were accompanied by damage of the vertebral bodies. Inclusion criteria included: (1) cases with neurological deficits and bone destruction of various degrees with little-to-no psoas or iliac abscess, (2) patients aged $>60$ years, (3) lesions confined to one segment or two adjacent segments, and (4) the absence of an extensive TB abscess. Patients who satisfied the surgical criteria were assigned to group A or group B: group A (16 cases) underwent a combined anterior and posterior approaches and group B (17 cases) underwent a posterior only approach. In fact, it is difficult to randomly select a surgical treatment method clinically. Therefore, in our study, cases in group A (combined anterior and posterior approach) were collected earlier, but cases in group B (posterior only) were collected in recent years.

All of the cases presented with constitutional symptoms, such as weakness, back pain, sweats, fever with weight loss and variable degrees of local deformity angle; the mean symptom duration was 6 months. None of the patients in this study had active lung TB or were HIV positive. Diagnosis was based on nonspecific laboratory findings and imaging results, including spinal radiographic films, computed tomography (CT) and magnetic resonance imaging. The neurological examination according to the American Spinal Injury Association impairment scale was grade B in 3 cases, C in 19 and D in 11. Initial and preoperative pain was assessed based on a visual analog scale. Most of the patients had multiple comorbidities, such as cardiovascular diseases, hepatitis B, diabetes mellitus and chronic obstructive pulmonary diseases (Table 1).

Sixteen patients who underwent anterior debridement, bone grafting and posterior instrumentation in a single- or two-stage procedure formed the control group (group A). The other 17 cases underwent single-stage posterior debridement, decompression, interbody fusion and instrumentation via a posterior only approach (group B). The same team reviewed the surgical indications and performed the operations.

\section{Preoperative procedure}

All patients received standard anti-TB chemotherapy $\left(300 \mathrm{mg} \mathrm{day}^{-1}\right.$ isoniazid, $450 \mathrm{mg} \mathrm{day}^{-1}$ rifampicin, $750 \mathrm{mg} \mathrm{day}^{-1}$ ethambutol, $750 \mathrm{mg} \mathrm{day}^{-1}$ pyrazinamide) for an average of 2-3 weeks prior to surgery. Surgery was postponed until anemia and hypoproteinemia recovered and there was a significant decrease in the erythrocyte sedimentation rate (ESR).

\section{Operative method}

In group B, posterior debridement, decompression, interbody fusion and instrumentation were performed in a single stage. The key surgical procedure

Table 1 Clinical data on the patients

\begin{tabular}{lcc}
\hline & Group A $(\mathrm{N}=16)$ & Group $B(\mathrm{~N}=17)$ \\
\hline Gender & 8 & 70 \\
Male & 8 & 7 \\
Female & & $70.5 \pm 5.1$ \\
& $69.8 \pm 4.2$ & \\
Age (years) & & 5 \\
& & 9 \\
Involved vertebrae & 4 & 2 \\
L3/4 & 8 & 1 \\
L4/5 & 3 & \\
L5/S1 & 1 & 2 \\
L3/4/5 & & 4 \\
Comorbidities & & 4 \\
Cardiovascular diseases & 5 & 6 \\
Diabetes mellitus & 3 & 2 \\
Hepatitis & 7 & \\
COPD & 6 & \\
None of the above diseases & 1 & \\
\hline
\end{tabular}

Abbreviation: COPD, chronic obstructive pulmonary disease. included decompression via the complete removal of the lesion through the healthy, bleeding bone from the posterolateral approach using curettes of various sizes and angles. We recommended reserving the insides of the pedicles for safety. We eliminated the pus and necrotic tissue by intraoperative pressurized washing (via a soft catheter inserted into the deep part of the lesion) and negative pressure suction. Correction of the local deformity was accomplished by installing contoured rods and exerting compression at middle anchoring point with cantilever bending maneuver to achieve local spinal alignment. For case with $\mathrm{S} 1 \mathrm{~TB}$, pedicle screws were inserted bilaterally at L5 and iliac wing, and entire vacuum in the S1 body was packed with bone block. Following fixation and correction, one or two cross links were installed to increase the stability of spine. Autologous or allograft particulate bone was implanted in the lateral diseased vertebral facet joints and between the transverse processes to promote bone fusion. Titanium mesh cages were not used in our series because of elderly patients usually with osteoporosis and in order to prevent cage subsidence and pseudarthrosis formation.

In group $\mathrm{A}$, posterior instrumentation without fusion was performed first, followed by anterior debridement and allografting in a single stage or two stages.

For patients with only the disc involved, transforaminal lumbar interbody fusion was carried out; for patients with destroyed vertebral bodies, partial corpectomy was performed. In both groups, suitable autogenous struts (allogeneic bone used in patients with severe osteoporosis) were transplanted from the ilium to the prepared bone trough to reconstruct the vertebrae. Streptomycin $(0.1 \mathrm{~g})$ and isoniazid $(0.2 \mathrm{~g})$ were administered locally; drainage and suturing of the incision were performed after the previously described procedures. The debrided material was sent for culture and histopathological examination.

\section{Postoperative procedure}

Typically, the drain was removed when drainage flow was $<50 \mathrm{ml}$ per $24 \mathrm{~h}$. Intravenous antibiotics were administered to prevent infection, and nutritional support was regularly performed. All patients were treated with the previously mentioned anti-TB chemotherapy regimen for 12-18 months postoperatively. Patients were allowed to gradually start walking with assistance from a plastic orthosis after remaining supine for an average of 2 weeks postoperatively, depending on their recovery of lower limb muscle strength. In the meantime, the bed was elevated at an angle of approximately $30^{\circ}$ to assist in expectoration and heart and lung function. The ESR and hepatic function were tested regularly.

\section{Follow-up index and statistical analysis}

Immediately after surgery, we obtained routine lateral and anteroposterior radiographs or CT to assess the extent of decompression and the placement of the graft and instrumentation. CT was also used to classify the degree of bony healing as clear fusion, delayed union or pseudarthrosis development according to the modified radiological criteria of Lee et al. ${ }^{12}$ (Table 2). The following indexes were recorded preoperativel and postoperatively and during the followup: (1) Cobb angle, (2) neurological status according to Frankel classification, (3) ESR, and (4) visual analog scale pain score. All statistical analyses were

Table 2 Modified criteria of Lee et al. ${ }^{12}$ for evaluating fusion radiologically

\begin{tabular}{ll}
\hline Grade & Description \\
\hline Definitive fusion & $\begin{array}{l}\text { Definitive bony trabeculae bridging across grafthost interface, } \\
\text { no movement }\left(<3^{\circ}\right) \text { on a flexion extension radiograph and no } \\
\text { gap at interface } \\
\text { No definitive trabeculae crossing but no detectable movement } \\
\text { Probable fusion }\end{array}$ \\
$\begin{array}{l}\text { Possible } \\
\text { pseudarthrosis }\end{array}$ & $\begin{array}{l}\text { No bony trabeculae crossing, no movement but identifiable } \\
\text { No traversing trabecular bone, definitive gap and } \\
\text { Definite }\end{array}$ \\
pseudarthrosis & movement $>3^{\circ}$
\end{tabular}


performed using SPSS 17.0 (SPSS, Inc., Chicago, IL, USA). We compared changes in laboratory and physical parameters in the two groups using the Student-Newman-Keuls test. Any discrepancy in normal distribution was analyzed using the rank-sum test; the significance level was 0.05 .

\section{RESULTS}

Table 1 shows a summary of the patients' data. All patients and back pain after surgery. Neurological status is shown in Table 3; no severe neurological complications were observed in either group.

Table 3 Neurological recovery according to the American Spinal Injury Association (ASIA) impairment scale (group A and group B)

\begin{tabular}{|c|c|c|c|c|c|c|c|c|c|c|c|}
\hline \multirow[t]{2}{*}{ Preoperation } & \multirow[t]{2}{*}{ Group $A / B$} & \multicolumn{5}{|c|}{$\begin{array}{l}\text { Final follow-up in } \\
\text { group } A\end{array}$} & \multicolumn{5}{|c|}{$\begin{array}{l}\text { Final follow-up in } \\
\text { group } B\end{array}$} \\
\hline & & $A$ & $B$ & $c$ & $D$ & E & $A$ & $B$ & C & $D$ & $E$ \\
\hline A & $0 / 0$ & & & & & & & & & & \\
\hline B & $1 / 2$ & & & & 1 & & & & & 2 & \\
\hline C & $10 / 9$ & & & & 2 & 8 & & & & 2 & 7 \\
\hline D & $5 / 6$ & & & & & 5 & & & & & 6 \\
\hline$E$ & $0 / 0$ & & & & & & & & & & \\
\hline
\end{tabular}
experienced significant improvement in constitutional symptoms

Table 4 Outcomes of two different surgical treatments for lower lumbar spinal TB in the aged

\begin{tabular}{lccc}
\hline & Group A (N=16) & Group B (N=17) & P-value \\
\hline Operation time (min) & $276.9 \pm 23.8$ & $193.8 \pm 22.5$ & $<0.05$ \\
Blood loss (ml) & $1187.5 \pm 163.0$ & $804.7 \pm 134.1$ & $<0.05$ \\
Hospitalization (days) & $18.2 \pm 3.2$ & $13.4 \pm 1.6$ & $<0.05$ \\
Duration of follow-up (months) & $41.4 \pm 4.2$ & $41.3 \pm 3.7$ & 0.954 \\
& & & \\
Cobb's angle( ${ }^{\circ}$ ) & & & \\
Preoperative & $18.8 \pm 6.3$ & $17.9 \pm 6.2$ & 0.714 \\
Postoperative & $2.4 \pm 6.1$ & $2.6 \pm 5.6$ & 0.942 \\
Angle correction & $16.3 \pm 2.0$ & $15.4 \pm 5.0$ & 0.068 \\
Final follow-up & $3.6 \pm 6.5$ & $4.0 \pm 5.7$ & 0.838 \\
Loss of correction & $1.1 \pm 0.7$ & $1.4 \pm 0.8$ & 0.287 \\
Angle lost rate (\%) & $6.5 \pm 5.5$ & $8.8 \pm 5.9$ & 0.258 \\
Fusion time (months) & $7.8 \pm 1.2$ & $8.4 \pm 1.6$ & 0.242 \\
& & & \\
ESR & & & \\
$\quad$ Preoperative & $74.6 \pm 10.6$ & $73.0 \pm 9.8$ & 0.664 \\
Final follow-up & $9.8 \pm 2.9$ & $9.5 \pm 3.0$ & 0.786 \\
& & & \\
VAS & & & 0.768 \\
Preoperative & $7.1 \pm 1.1$ & $7.2 \pm 1.1$ & 0.577 \\
Final follow-up & $1.1 \pm 0.9$ & $1.2 \pm 0.8$ & \\
\hline Abbreviations. ESR, erythrocyte sedimentation rate. TB, tuberculosis. vAS, visual analog scale.
\end{tabular}
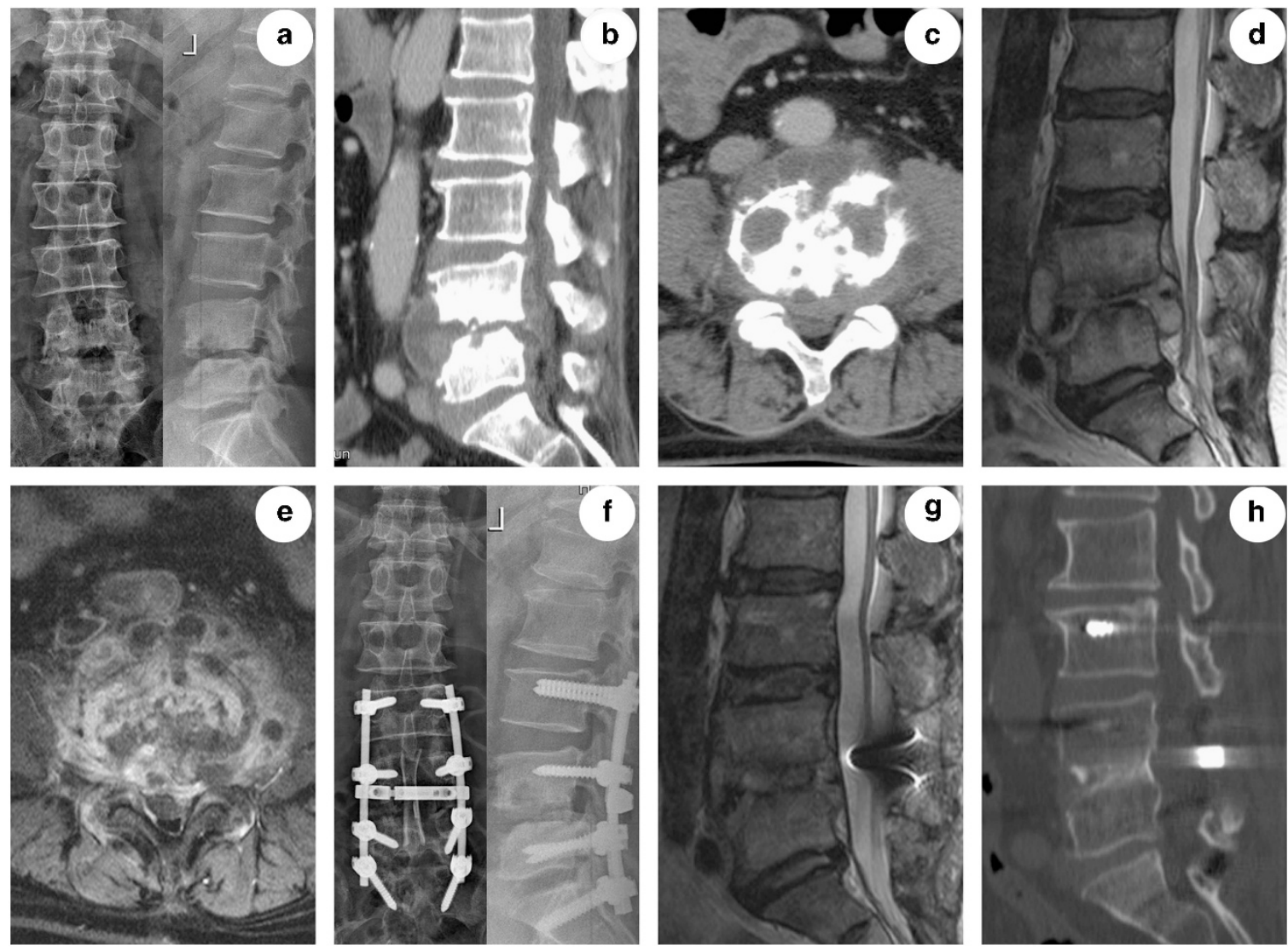

Figure 1 (a) A preoperative X-ray of a 65-year-old male demonstrated the destruction of L4-5, with a Cobb angle of $-16^{\circ}$. CT and magnetic resonance imaging (MRI) (b-e) show vertebral bone destruction, paravertebral abscess formation and spinal cord compression. (f) Postoperative radiography showed that fixation was in good position, kyphosis had improved significantly, and the Cobb angle was $-5^{\circ}$. (g) Postoperative MRI showed spinal cord compression was decompressed. (h) At 43-month follow-up, solid bone fusion and satisfactory sagittal alignment of lumbar spine were obtained. 
The overall mean follow-up was 41.3 months (range 36-48 months). The ESR, visual analog scale score, surgical duration, blood loss and average hospital stay are recorded in Table 4 . The average operative duration, blood loss and hospital stay for group B were significantly less than the corresponding values for group A $(P<0.05$; Table 4$)$. All grafted bones in group A ultimately fused, with fusion times ranging from 6 to 10 months (mean 7.8 months). In group B, all cases except for one achieved fusion, with fusion times ranging from 6 to 12 months (mean 8.4 months) (Figures 1 and 2). There were significant differences $(P<0.05)$ between the preoperative and postoperative Cobb's angle in both groups. There were no significant differences $(P>0.05)$ between the groups in terms of the postoperative Cobb's angle, angle correction, loss of correction or angle loss rate (Table 4). Plastic orthosis wearing time and fusion time were longer, and loss of correction was somewhat greater in patients who had partial corpectomy carried out than in those who had transforaminal lumbar interbody fusion carried out.

Four patients in group A experienced operative complications. One incision was superficially infected with Escherichia coli and was successfully cured with antibiotics. One patient who suffered from fistula formation 1 week postoperatively was healed by weekly local isoniazid therapy. One patient with chronic obstructive pulmonary disease on admission suffered from breathing infections, which were treated successfully with antibiotics. One patient experienced anti-TB drug-induced liver dysfunction. This patient received modified
anti-TB treatment and hepatoprotective treatment until liver function recovered.

In group $\mathrm{B}$, fusion was delayed in one patient because of a psoas abscess observed at the 6-month follow-up and caused by irregular anti-TB treatment; the abscess was successfully treated via minimally invasive CT-guided percutaneous catheter drainage and regular chemotherapy.

\section{DISCUSSION}

Despite ongoing efforts by the World Health Organization and local health authorities, TB is still prevalent in some developing countries and affects all susceptible individuals, including the aged. ${ }^{13}$ Because of decreased body resistance and poor physical function, TB presentation can be atypical in older adults and may be misdiagnosed as intervertebral disc degeneration, deformity, spinal stenosis and neoplasia, thus delaying accurate diagnosis and treatment. ${ }^{14}$ Because symptoms of spinal TB in the elderly are generally mild and concealed in the early stages and may not be accompanied by significant changes in the ESR, the diagnosis of TB is difficult in this patient population, especially in remote areas with limited medical facilities. ${ }^{15}$ The incidence of nerve damage from spinal TB has been reported to be much higher in older adults compared with the other groups. ${ }^{16}$

Because elderly patients often have multiple chronic diseases and poor health status, conservative treatment with anti-TB chemotherapy has been applied in most cases to avoid surgical risk and associated
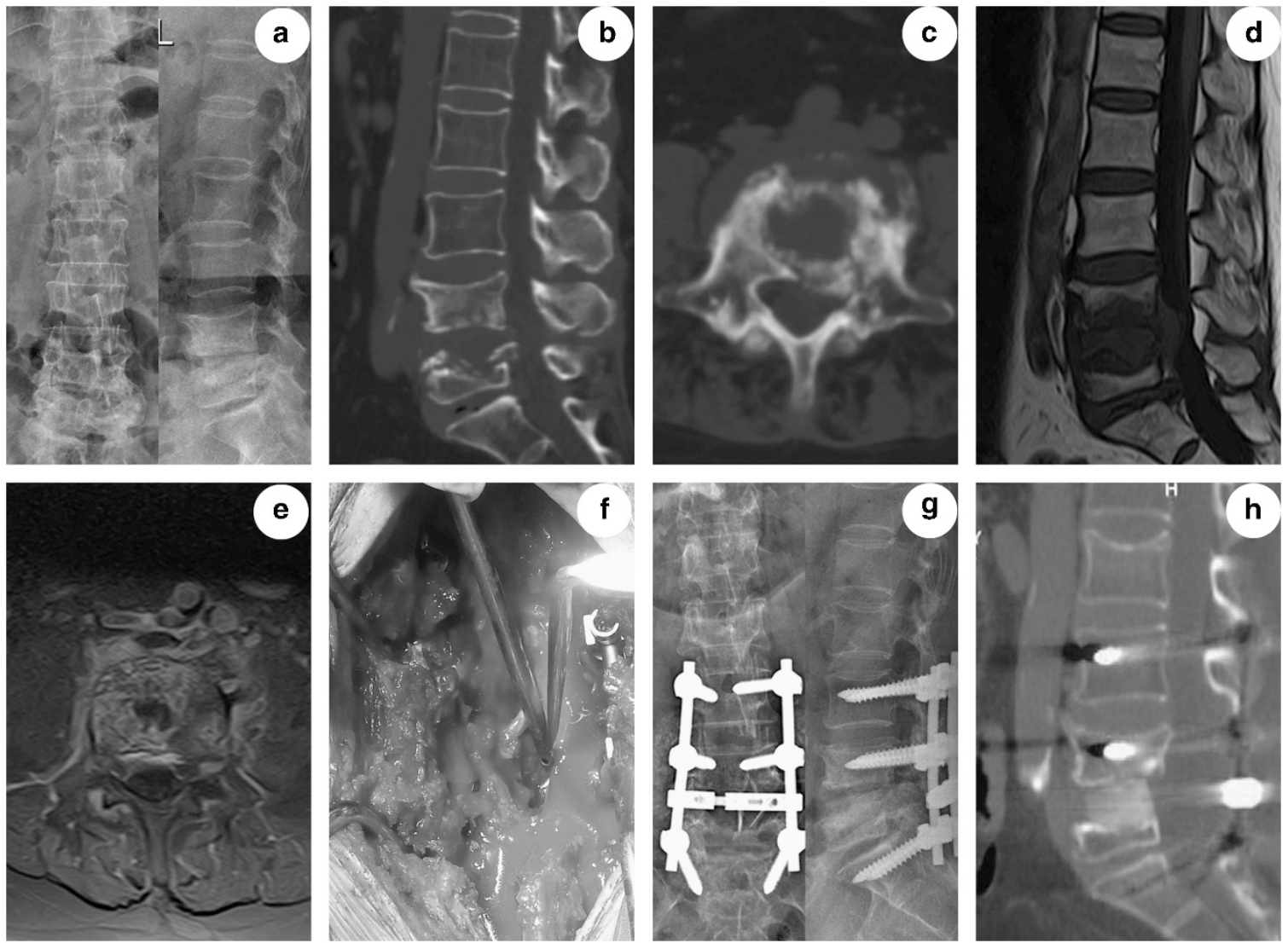

Figure 2 (a) A preoperative X-ray of a 74-year-old female demonstrated the destruction of L4-5, with a Cobb angle of $-25^{\circ}$. CT and magnetic resonance images (b-e) show vertebral bone destruction, paravertebral abscess formation and spinal cord compression. (f) Intraoperative photo of posterior debridement, decompression, interbody fusion and instrumentation via single stage. Postoperative radiography (g) showed that fixation was in good position, kyphosis had improved significantly, and the Cobb angle was $-10^{\circ}$. (h) At 40-month follow-up, solid bone fusion and satisfactory sagittal alignment of lumbar spine were obtained. 
complications, resulting in low fusion rates and high recurrence rates. ${ }^{16}$ Additionally, for cases with nerve compression secondary to significant vertebral body damage, conservative treatment requires prolonged bed rest, which can lead to serious complications strongly affecting patients' quality of life. Moreover, the mortality of older adults is triple than that of younger adults during therapy. ${ }^{17}$ Finally, tuberculous foci may be surrounded by a hardened bone wall that forms a huge barrier against the penetration of anti-TB drugs into the lesion, resulting in failure of TB bacterium eradication. For these reasons, surgical treatment still has an important role in the management of lower lumbar TB with neurological deficits in the aged. The choice of anterior or posterior surgical approach is controversial. ${ }^{18}$ As of yet, no studies have compared the outcomes between the posterior only approach and the posterior plus anterior approach for lower lumbar spinal TB with neurological deficits in the elderly.

Some authors ${ }^{18,19}$ have previously proposed combined anterior and posterior surgery for treating lower lumbar TB. Although this approach can achieve complete debridement and firm fixation, it is still controversial. In addition to the limitations inherent with the anterior approach, aged patients often have comorbidities, such as diabetes, cardiovascular disease and respiratory disease. As a consequence, the large surgical trauma and high risk associated with a single-stage combined approach pose challenges to both patients and surgeons. Meanwhile, a two-stage operation increases hospital stays and costs and also requires patients to stay in bed for a longer period of time after surgery, increasing the incidence of complications.

The 17 cases in group B underwent single-stage posterior debridement, decompression, interbody fusion and instrumentation via a posterior only approach, which facilitated better outcomes than those experienced by group A patients, who underwent a combined posterior and anterior surgical approach. Compared with other surgical methods, we believe that the posterior only approach has the following advantages. First, this approach uses pedicle screws, which provide three-dimensional correction and stabilization that is much stronger than that achieved by anterior instrumentation, making this technique especially suitable for elderly patients with varying degrees of osteoporosis. Second, lesion debridement, decompression and fixation are completed at one time, reducing surgery time, the amount of bleeding and the risk of nerve and major vascular injury. Third, the involved anatomy is relatively simple, avoiding possible injury of the large blood vessels, nerves and ureters located near the lower lumbar spine as well as invasion of the paraspinal soft tissues affected by the anterior approach.

For cases with a failed anterior column, the controversy surrounding the use of a posterior only approach to treat spinal TB involves whether such an approach can completely accomplish focal debridement and whether reconstruction of spinal stability can be effectively obtained. The posterior only approach offers no advantage in terms of debridement; however, with the development of effective anti-TB drugs, tuberculous lesions may heal through spontaneous fusion, and complete debridement is not unduly emphasized. ${ }^{15}$ In the present study, all cases in both groups ultimately healed with good bone fusion. The correction of Cobb's angle was $15.4 \pm 5.0^{\circ}$ in group B patients, and was effective in maintaining correction, with an average loss of $1.4 \pm 0.8^{\circ}$, which is similar to the data reported in the literature. ${ }^{19,20}$ Accordingly, in our study, the posterior approach achieved equally good results compared with the combined approach in terms of deformity correction and bone fusion.

The posterior only approach has limits. It cannot be used for cases with a wide range of $\mathrm{TB}$ abscesses and lesions or with multiple involved levels extending to the upper lumbar spine, in which case an anterior debridement is necessary. Indications for this surgery can be summarized as follows: (1) lesions are confined to one segment or two adjacent segments, (2) the patient has undergone several anterior operations and the anatomical structure is unclear, (3) the lesion area does not need long segmental bone grafting after debridement to restore the height of the spine, (4) there are not extensive TB abscesses or lesions that could be thoroughly debrided during the preoperative evaluation, and (5) lower lumbar TB with deformity in which favorable correction cannot be achieved merely through an anterior approach.

Each case of lower lumbar TB in the aged needs to be considered individually, because controversy remains as to the optimal treatment. In our study, the clinical and radiographic results of the patients who underwent a posterior only approach were good, but these are preliminary results in a small group of patients with a relatively short follow-up for some patients. Further study with a larger number of patients and longer follow-up is necessary.

\section{CONCLUSION}

Surgical management via single-stage posterior debridement, decompression, interbody fusion and instrumentation can be an effective treatment for lower lumbar tuberculous spondylitis with neurological deficits in the aged. This method can effectively reduce complications, improve neurological function, reconstruct spinal stability and reduce trauma.

\section{DATA ARCHIVING}

There were no data to deposit.

\section{CONFLICT OF INTEREST}

The authors declare no conflict of interest.

\section{ACKNOWLEDGEMENTS}

This publication was partially funded by the National Natural Science Foundation of China (81171736).

1 Peto HM, Pratt $\mathrm{RH}$, Harrington TA, LoBue PA, Armstrong LR. Epidemiology of extrapulmonary tuberculosis in the United States, 1993-2006. Clin Infect Dis 2009, 49: 1350-1357.

2 Maeda Y, Izawa K, Nabeshima T, Yonenobu K. Tuberculous spondylitis in elderly Japanese patients. J Orthop Sci 2008; 13: 16-20.

3 Talu U, Gogus A, Ozturk C, Hamzaoglu A, Domanic U. The role of posterio instrumentation and fusion after anterior radical debridement and fusion in the surgical treatment of spinal tuberculosis: experience of 127 cases. J Spinal Disord Tech 2006; 19: 554-559.

4 Medical Research Council Working Party on tuberculosis of the spine. A controlled tria of anterior spinal fusion and debridement in the surgical management of tuberculosis of the spine in patients on standard chemotherapy: a study in two centres in South Africa. Seventh Report of the Medical Research Council Working Party on tuberculosis of the spine. Tubercle 1978; 59: 79-105.

5 Lifeso RM, Weaver P, Harder EH. Tuberculous spondylitis in adults. J Bone Joint Surg Am 1985; 67: 1405-1413.

6 Rajasekaran S, Shanmugasundaram TK, Prabhakar R, Dheenadhayalan J, Shetty AP, Shetty DK. Tuberculous lesions of the lumbosacral region. A 15-year follow-up of patients treated by ambulant chemotherapy. Spine (Phila Pa 1976) 1998; 23: 1163-1167.

7 Lehmer SM, Steffee AD, Gaines RJ. Treatment of L5-S1 spondyloptosis by staged L5 resection with reduction and fusion of L4 onto S1 (Gaines procedure). Spine (Phila Pa 1976) 1994; 19: 1916-1925.

8 Zaveri GR, Mehta SS. Surgical treatment of lumbar tuberculous spondylodiscitis by transforaminal lumbar interbody fusion (TLIF) and posterior instrumentation. J Spinal Disord Tech 2009; 22: 257-262.

9 Hirakawa A, Miyamoto K, Masuda T, Fukuta S, Hosoe H, linuma N et al. Surgical outcome of 2-stage (posterior and anterior) surgical treatment using spinal instrumentation for tuberculous spondylitis. J Spinal Disord Tech 2010; 23: 133-138.

10 Zevallos M, Justman JE. Tuberculosis in the elderly. Clin Geriatr Med 2003; 19 $121-138$ 
11 Gong K, Wang Z, Luo Z. Single-stage posterior debridement and transforaminal lumbar interbody fusion with autogenous bone grafting and posterior instrumentation in the surgical management of lumbar tuberculosis. Arch Orthop Trauma Surg 2011; 131: 217-223.

12 Lee CK, Vessa P, Lee JK. Chronic disabling low back pain syndrome caused by internal disc derangements. The results of disc excision and posterior lumbar interbody fusion. Spine (Phila Pa 1976) 1995; 20: 356-361.

13 Zhang $H Q$, Lin MZ, Shen KY, Ge L, Li JS, Tang MX et al. Surgical management for multilevel noncontiguous thoracic spinal tuberculosis by single-stage posterior transforaminal thoracic debridement, limited decompression, interbody fusion, and posterior instrumentation (modified TTIF). Arch Orthop Trauma Surg 2012; 132: 751-757.

14 Mori T, Leung CC. Tuberculosis in the global aging population. Infect Dis Clin North Am 2010; 24: 751-768.

15 Pande KC, Babhulkar SS. Atypical spinal tuberculosis. Clin Orthop Relat Res 2002; 398: $67-74$
16 Moon MS. Tuberculosis of the spine. Controversies and a new challenge. Spine (Phila Pa 1976) 1997; 22: 1791-1797.

17 Pratt RH, Winston CA, Kammerer JS, Armstrong LR. Tuberculosis in older adults in the United States, 1993-2008. J Am Geriatr Soc 2011; 59: 851-857.

18 Jain AK, Dhammi IK, Prashad B, Sinha S, Mishra P. Simultaneous anterior decompression and posterior instrumentation of the tuberculous spine using an anterolateral extrapleural approach. J Bone Joint Surg $\mathrm{Br}$ 2008; 90: 1477-1481.

19 Zhang HQ, Li JS, Zhao SS, Shao YX, Liu SH, Gao Q et al. Surgical management for thoracic spinal tuberculosis in the elderly: posterior only versus combined posterior and anterior approaches. Arch Orthop Trauma Surg 2012; 132: 1717-1723.

20 Guven O, Kumano K, Yalcin S, Karahan M, Tsuji S. A single stage posterior approach and rigid fixation for preventing kyphosis in the treatment of spinal tuberculosis. Spine (Phila Pa 1976) 1994; 19: 1039-1043. 